\title{
Research on Virtual Network Remapping of Demand Change
}

$$
\text { Yiqiu Fang }{ }^{1, a} \text {, Hongjian } \mathrm{Wu}^{2, * b}
$$

\author{
${ }^{1}$ School of Computer Science and Technology, Chongqing University of Posts and \\ Telecommunications, Chongqing 400000, China; \\ ${ }^{2}$ School of Computer Science and Technology, Chongqing University of Posts and \\ Telecommunications, Chongqing 400000, China.
}

Corresponding author Email: afangyq@cqupt.edu.cn bdudoulion@163.com

\begin{abstract}
Keywords: Virtual network, Remapping,Virtual network mapping.
\end{abstract}
\begin{abstract}
Virtual network request resources are dynamically changing, and there are fewer studies in this area.In view of this situation, in this paper the virtual network mapping model is improved, the model of minimizing the cost of resource request change and improving the access rate of virtual network is established, and based on the DVNMA algorithm, the resource reuse strategy is used to improve it.The simulation results show that the algorithm reduces the cost of remapping and obtains the high virtual network request acceptance rate.
\end{abstract}

\section{Introduction}

At present in our real daily life, the Internet has played a very important role.However, the purpose of the existing Internet at the beginning of the design is to provide researchers with data communication functions such as information exchange, text transmission and remote access. The network control model and the easy-to-access design principle The development of the Internet has brought convenience, but in the face of the current Internet mass data transmission needs and large-scale business application environment is exposed to the current Internet architecture inherent some drawbacks.

At present, most of the research is only for the virtual network request is a fixed situation design effective virtual network mapping algorithm for dynamic virtual network request mapping algorithm is still relatively small.

Some authors propose a distributed autonomous virtual resource allocation algorithm in which the physical node can identify the overload traffic of a physical link and migrate as much as possible through the virtual node to reduce the number of hops of the physical link. Mechanism does not give the method of migrating virtual nodes [1].Some scholars have proposed reconfiguration methods for dynamic virtual network requests; however, this approach only considers how to reconfigure the VNs at a minimum cost when a running $\mathrm{VN}$ request changes [2].In addition, the researchers consider the dynamic resource allocation problem in the virtual network mapping from the perspective of workload probability, set a tolerance threshold for each virtual network, establish a two-phase virtual network mapping algorithm, and then map the virtual network node in a shared way, The free resource block on the node / link is shared by all the virtual nodes / links, and when the resource is saturated, the greedy algorithm is used to find the candidate mapping target for the virtual node link that does not meet the demand [3].In addition, the virtual network mapping problem of hybrid data center is modeled by nonlinear optimization theory, and then the improved greedy algorithm is used to solve the problem of virtual hybrid network.But did not consider the problem of resource changes that have mapped virtual networks [4].

\section{The MILP method}

In this paper, the MILP method is used to solve the problem of optimal optimization of dynamic VN requests into mathematical optimization problems.Similar to the literature [5], the following three tables give the main symbols used in the system model and their meanings. 
Table 1 Set Definitions

\begin{tabular}{cc}
\hline & Sets \\
\hline $\mathrm{V}^{\mathrm{S}}$ & Collection of the Underlying Physical Network \\
$\mathrm{E}^{\mathrm{s}}$ & Collection of the Underlying Physical Network \\
$\mathrm{P}^{\mathrm{S}}$ & Collection of the Underlying Physical Network \\
Path & Collection of the Original VN Requests the \\
$\mathrm{V}_{1}{ }^{\mathrm{V}}$ & Node \\
$\mathrm{V}_{2}{ }^{\mathrm{v}}$ & Collection of the Current VN Requests the \\
$\mathrm{E}_{2}{ }^{\mathrm{V}}$ & Collection of the Current VN Requests the Link \\
$\mathrm{V}_{\mathrm{r}}{ }^{V}$ & Collection of the VN Nodes Which Need to \\
& Reallocate Resources \\
\hline
\end{tabular}

Table 2 Constant Definitions

\begin{tabular}{|c|c|c|}
\hline Parameter & Meaning & Range \\
\hline$\beta$ & Allocation of Resources & $\geq 0$ \\
\hline$\phi_{\mu v}$ & $\begin{array}{l}\text { Original VN Mapping } \\
\text { Relationship }\end{array}$ & $\in(0,1)$ \\
\hline $\operatorname{capa}(v)$ & $\begin{array}{l}\text { The Physical Capacity of the } \\
\text { Underlying Physical Node v }\end{array}$ & $\geq 0$ \\
\hline $\operatorname{capa}(e)$ & $\begin{array}{l}\text { The Physical Capacity of The } \\
\text { Underlying Physical Link e }\end{array}$ & $\geq 0$ \\
\hline req $(u)$ & $\begin{array}{l}\text { VN Node u Resource } \\
\text { Requirements }\end{array}$ & $\geq 0$ \\
\hline$b(e)$ & $\begin{array}{l}\text { VN Link e Resource } \\
\text { Requirements }\end{array}$ & $\geq 0$ \\
\hline$d(e)$ & $\begin{array}{l}\text { The Transmission Delay } \\
\text { Constraint of the VN Link e }\end{array}$ & $\geq 0$ \\
\hline realloc $(u)$ & Reassignment of Resources & $\in(0,1)$ \\
\hline
\end{tabular}

Table 3 Constant Definitions

\begin{tabular}{cll}
\hline Variables & \multicolumn{1}{c}{ meaning } & Range \\
\hline $\mathrm{h}_{\mu \nu}$ & The Mapping of the Current VN $\in(0,1)$ \\
$r l(e)$ & $\begin{array}{l}\text { The Amount of Resources } \geq 0 \\
\text { Provided by the Physical Link e } \\
\text { The Amount of Bandwidth } \geq 0\end{array}$ \\
Delay $(p)$ & $\begin{array}{l}\text { Resources Allocated for Path p } \\
\text { The Propagation Delay of Path } \geq 0\end{array}$ \\
$\mathrm{M}_{\mathrm{E}}(e)$ & $\begin{array}{l}\text { P The Physical Path of the VN } \in \mathrm{P}^{S} \\
\text { Link e }\end{array}$ \\
\hline
\end{tabular}

\subsection{Problem Definition.}

The questions to be studied can be defined as follows.A known:Given the underlying infrastructure in equation (1),the original virtual network mapping in equation (2),the current virtual network mapping in equation (3). 


$$
\begin{gathered}
\mathrm{G}^{s}=\left(V^{s}, E^{s}, C_{V}^{s}, C_{E}^{s}\right) \\
\mathrm{OVN}_{G_{1}^{V}}=\left(V_{1}^{V}, E_{1}^{V}, R_{1}^{V}, R_{1}^{E}\right) \\
\operatorname{EVN}_{G_{2}^{V}}=\left(V_{2}^{V}, E_{2}^{V}, R_{2}^{V}, R_{2}^{E}\right)
\end{gathered}
$$

\subsection{Restrictions.}

In the $\mathrm{VN}$ mapping process, must meet a variety of constraints,Including resource capacity constraints, constraints of mapping location selection, delay constraints for transmission paths, and so on[6].Since the mapping of nodes is a one-to-one mapping, equation (4) ensures that a VN node is mapped to a physical node.

$$
\sum_{v \in V^{s}} h_{\mu v}=1 \quad \forall u \in V_{2}^{V}
$$

Constraints (5) ensure that any one of the physical nodes deployed by the same number of VN nodes no more than 1

$$
\sum_{\mu \in \mathrm{V}_{2}^{V}} h_{\mu \nu} \leq 1 \quad \forall v \in V^{S}
$$

Constraints (6) Ensure that the amount of CPU resources provided by any node must not exceed the total capacity of its own resources.

$$
r n(v) \leq \operatorname{capa}(v), \forall v \in V^{S}
$$

Constraints (7) Ensure that the transmission delay of the physical path of each VN link does not exceed the upper limit of the acceptable delay for the VN link.

$$
\operatorname{Delay}\left(\mathrm{M}_{E}(e)\right) \leq \mathrm{d}(\mathrm{e}), \forall \mathrm{e} \in \mathrm{E}_{2}^{V}
$$

Equations (8 and Constraints (9) are physical link capacity constraints that ensure that the amount of resources provided by each physical link must not exceed its total total amount of resources[7].

$$
\begin{aligned}
& r l(e)=\sum_{f \in E_{2}^{V}, e \in M_{E}(f)} \operatorname{Band}\left(M_{E}(f)\right), \forall e \in E^{S} \\
& r l(e) \leq \operatorname{capa}(e), \forall e \in E^{S}
\end{aligned}
$$

\subsection{Optimize the Target.}

The cost function for mapping the newly added VN nodes and VN links is shown in equation (10).

$$
\begin{aligned}
& \text { Cost }_{\text {add }}=\sum_{\mu \in\left(V_{2}^{V}-V_{1}^{V}\right) v v \in V^{s}} r e q(u) \bullet h_{u v} \bullet \cos t(v) \\
& +\sum_{e \in\left(E_{2}^{V}-E_{1}^{V}\right)} \sum_{f \in M_{E}(e)} \frac{\varepsilon}{\operatorname{capa}(f)+\delta} \bullet b(e) \bullet \cos t(f)
\end{aligned}
$$

Remodeling costs for $\mathrm{VN}$ components is shown in equation (11).

$$
\begin{aligned}
& \text { Cost }_{\text {rem }}=\sum_{e \in V_{\text {rem }}} \sum_{v \in V^{s}}\left(\operatorname{req}_{t}(u)-\operatorname{req}_{t-1}(u)\right) \bullet h_{u v} \bullet \cos t(v) \\
& +\sum_{e \in E_{\text {rem }}} \sum_{f \in M_{E}(e)} \frac{\varepsilon}{\operatorname{capa}(f)+\delta} \bullet b(e) \bullet \cos t(f)
\end{aligned}
$$

Where A and B represent the VN node and the VN link set that need to be mapped, respectively. VN component reconfiguration costs is shown in equation (12)[8].

$$
\text { Cost }=\text { Cost }_{\text {add }}+\text { Cost }_{\text {rem }}
$$

Objective function is shown in equation (13).

$$
\text { Minimize Cost }
$$


The acceptance rate of the virtual network request represents the probability that the virtual network request is accepted at time $t$.

$$
\text { AcceptRatio }=\lim _{\mathrm{T} \rightarrow \infty}\left(\sum_{t=0}^{T} V N R A\right) /\left(\sum_{t=0}^{T} V N R\right)
$$

Where VNRA represents the number of virtual network requests successfully mapped by the physical network, and VNR represents the total number of virtual network requests arriving at time $\mathrm{t}[9]$.

\subsection{DVNMA_R algorithm}

The optimal VN mapping problem is an NP-hard problem, and the algorithm design is more difficult, so the heuristic algorithm is designed[10]. At the same time, in order to take into account the difficulty of simulation and practical solution to the problem.In the simulation, AC, DC, DRR, IRR scene is a group of appear.

In the process of adding physical links, for a task in the re-allocation of resources in two steps to achieve.The first step remaps the associated VN components, where the associated VN nodes are mapped to other physical nodes with sufficient CPU resources,And then move the task to its corresponding $\mathrm{VN}$ node where the new physical node to continue[11].Before starting the remapping of the VN nodes, the VN nodes that need to be remapped are ranked in descending order of the resource requirements and stored in the queue Q1. For each node $\mathrm{v}$ in the queue Q1, the VN node $\mathrm{v}$ And their associated VN links are mapped to other physical nodes and physical paths that are adequately available.And then use the shortest path algorithm, between the old and new physical nodes to calculate a bandwidth resource sufficient shortest path for the task of migration.

In the process of adding a physical node, the user needs to submit to the InP a VN node or VN link request for resource needs before the new task[12]. The new VN node request to be mapped is first placed in descending order of resource requirements and stored in queue Q2. For the node v in each queue Q2, the VN node $\mathrm{v}$ and the VN link associated with $\mathrm{v}$ are mapped to the physical node and the physical link with sufficient resources at the minimum cost.If the above operation is not completed within five units of time, the remapping will be placed at the end of the task for processing, in order to increase the access time per unit time.

\section{Simulation and Analysis}

\subsection{Simulation Environment and Parameter Settings.}

In the simulation experiment, use the GT-ITM tool to generate the topology of the physical network and the topology of the virtual network[13].First, create the underlying network and enter all the virtual requests and put a queue, will be embedded in the virtual network in the process of a pop-up of each virtual request.The purpose of the simulation is to verify that the algorithm can achieve a joint goal:1) to improve the unit time within the virtual network request access rate,2) effectively reduce the virtual network request changes caused by the overhead.Virtual network requests are subject to random even distribution.

Table 4 Parameter Settings

\begin{tabular}{ccccc}
\hline Topology & $\begin{array}{c}\text { Number of } \\
\text { Nodes }\end{array}$ & Connectivity & $\begin{array}{c}\text { Node } \\
\text { Capacity }\end{array}$ & $\begin{array}{c}\text { Bandwidth } \\
\text { Capacity }\end{array}$ \\
\hline $\begin{array}{c}\text { Physical } \\
\text { network }\end{array}$ & $60 \sim 100$ & 20 & 100 unit & 100 unit \\
$\begin{array}{c}\text { Virtual } \\
\text { network }\end{array}$ & $4 \sim 20$ & 50 & $0 \sim 20$ unit & $0 \sim 50$ unit \\
\hline
\end{tabular}

\subsection{Simulation Results and Analysis.}

In Experiment 1, it is assumed that the arrival of the virtual network request follows the Poisson distribution,there are four virtual network requests in 100 time units, and the number of physical network nodes is set to 60, 70, 80, 90 and 100 respectively.Line 200 virtual network requests, each case running 30 times to take the average,experimental results as shown in Fag 1,It can be seen from the figure DVNMA_R algorithm cost less than DVNMA.This is because the DVNMA_R algorithm 
prioritizes the virtual network request to reduce the resources, so that the subsequent network request mapping is sufficient resources.Using DVNMA mapping virtual network request can choose to increase resources,and DVNMA_R algorithm uses a node reusable technology,So that the new nodes have little effect on the mapping result using DVNMA_R algorithm.

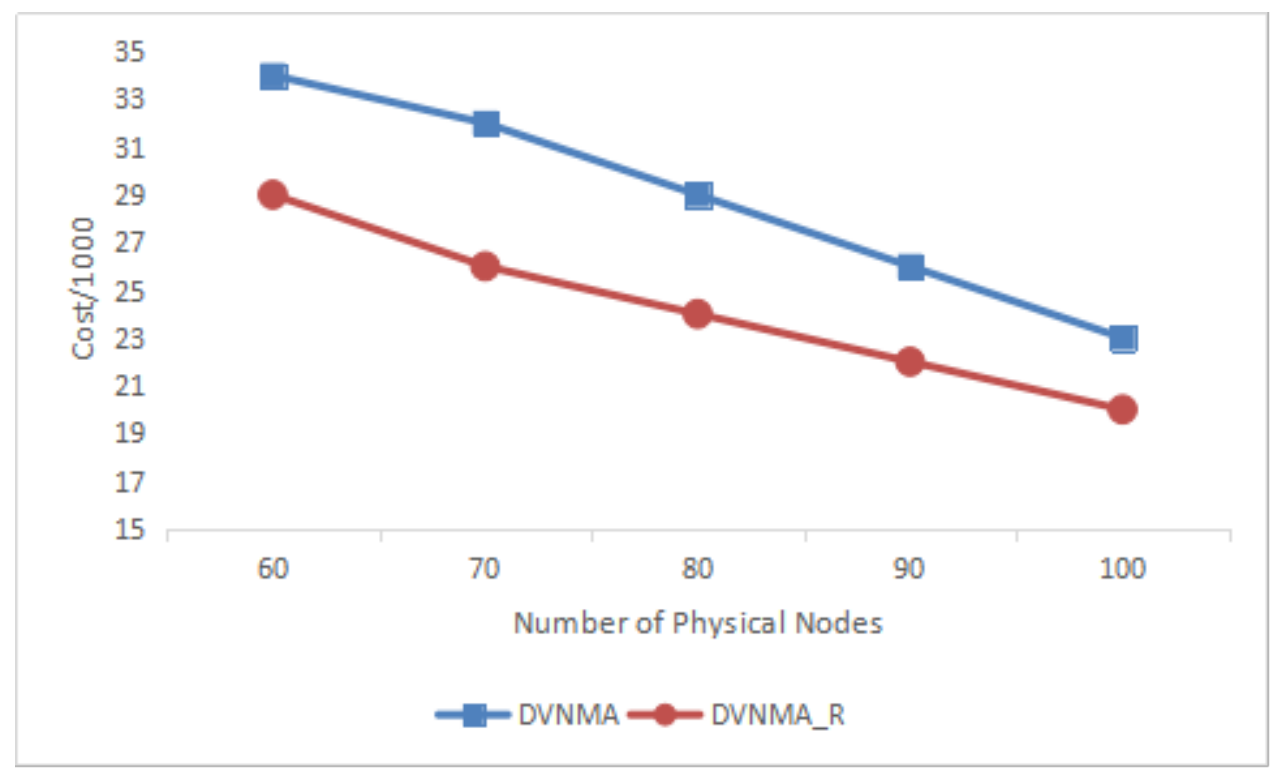

Fig. 1 Virtual network mapping costs for different physical nodes

In Experiment 2, the number of physical network nodes is set to 80.Run 1000 virtual network requests, run 30 times to take the average, the experimental results shown in Figure 23.It can be seen from Figure 2 that the acceptance rate of the two algorithms in the previous 2000s is drastically decreased due to the gradual arrival of the virtual network as the virtual network arrives, and the ability to carry new virtual network requests is weakened.With the increase of time, the acceptance rate of the algorithm tends to be stable and the receiving rate of DVNMA_R algorithm is about 3\% higher than that of DVNMA.The reason is that the algorithm uses reusable technology, which can save part of the link mapping overhead, can make the physical network to carry more virtual network requests.So that the virtual network request to take up resources to meet the changes as soon as possible to complete the task to complete the task, and not the success of the map is not directly added to the queue behind the sliding window waiting for a period of time and then remapping, thus improving the virtual network to complete the work effectiveness.In the mapping adjustment process is mapped to meet the requirements and the remaining resources of the largest node, so to a certain extent, although the increase in mapping costs, but the load tends to balance, for the subsequent arrival of the virtual network request to provide more mapping success opportunity. 


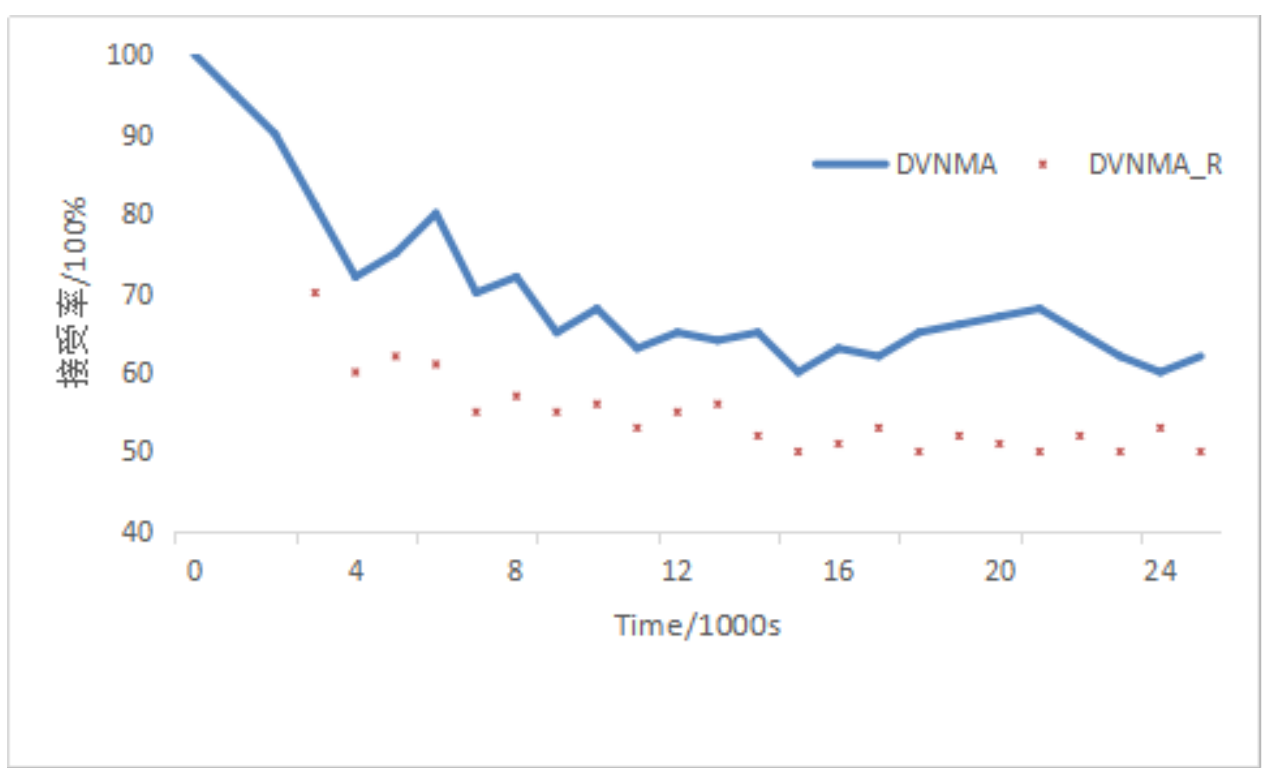

Fig. 2 The acceptance rate of virtual network requests

\section{Summary}

This paper studies the virtual network request mapping problem which is closer to the actual situation, and classifies the virtual network request.The DVNMA algorithm is improved and the established model can well reflect the performance of the algorithm.At the same time in the simulation process, also adopted a more reasonable alternative to simplify the simulation experiment.Experimental results show that DVNMA_R algorithm has some improvement on the basis of DVNMA algorithm.However, the virtual network mapping problem is always an NP-hard problem, follow-up based on the actual situation of the study is essential.

\section{References}

[1]. Marquezan C C, Nobre J C, Granville L Z, et al. Distributed Reallocation Scheme for Virtual Network Resources[J]. 2009:1-5.

[2]. Sun G, Yu H, Anand V, et al. A cost efficient framework and algorithm for embedding dynamic virtual network requests[J]. Future Generation Computer Systems, 2013, 29(5):1265 - 1277.

[3]. Mao Y X, Guo F Y, Hu C H, et al. Sharing Based Virtual Network Embedding Algorithm With Dynamic Resource Block Generation[J]. IEEE Communications Letters, 2015, 19(12):2126-2129.

[4]. Ahmed A U, Islam M T, Ismail M, et al. Dynamic resource allocation in hybrid access femtocell network.[J]. Thescientificworldjournal, 2014, 2014(3):539720.

[5]. Nakano H, Seki S, Kageyama H. Generalized and Resource-Efficient VNet Embeddings with Migrations[J]. Arxiv, 2010, 12(28):7772-7774.

[6]. Wang Y, Keller E, Biskeborn B, et al. Virtual routers on the move:live router migration as a network-management primitive[C]// ACM SIGCOMM 2008 Conference on Applications, Technologies, Architectures, and Protocols for Computer Communications, Seattle, Wa, Usa, August. DBLP, 2008:231-242.

[7]. Wang Y, Merwe J V D, Rexford J. VROOM: Virtual routers on the move[J]. Acm Sigcomm Computer Communication Review, 2008, 38(4).

[8]. Loureiro D, Goncalves P, Nogueira A. NETCONF agent for link state monitoring[C]// IEEE International Conference on Communications. IEEE, 2012:6565-6569. 
[9]. Lepiarz M, Onderka Z. Agent System for Load Monitoring of the Heterogeneous Computer Network[M]// Parallel Processing and Applied Mathematics. Springer Berlin Heidelberg, 2001:364-368.

[10]. Marquezan C C, Granville L Z, Nunzi G, et al. Distributed autonomic resource management for network virtualization[C]// IEEE/IFIP Network Operations and Management Symposium, NOMS 2010, 19-23 April 2010, Osaka, Japan. DBLP, 2010:463-470.

[11]. Lee K H, Hui P M. High-performance distribution of limited resources via a dynamical reallocation scheme[J]. Physica A Statistical Mechanics \& Its Applications, 2008, 387(387):6657-6662.

[12]. Marquezan C, Nunzi G, Brunner M. Method for operating at least one virtual network on a substrate network and a virtual network environment: US, US8745211[P]. 2014.

[13]. Zhu J. Benchmarking Virtual Network Mapping Algorithms[J]. 2012. 